\title{
Interaction of Ethyl Chloride with Amorphous Solid Water Thin Film on Ru(001) and O/Ru(001) Surfaces ${ }^{\dagger}$
}

\author{
Yousif Ayoub and Micha Asscher* \\ Institute of Chemistry and the Farkas Center for Light Induced Processes, \\ The Hebrew University of Jerusalem, Jerusalem 91904, Israel
}

Received: January 30, 2009; Revised Manuscript Received: April 11, 2009

\begin{abstract}
The adsorption of ethyl chloride (EC) on clean and oxygen covered $\mathrm{Ru}(001)$ surfaces and its interaction with coadsorbed amorphous solid water (ASW) is reported based on temperature-programmed desorption (TPD) and work function change $(\Delta \Phi)$ measurements. Adsorption of EC is characterized by a decrease of $2.1 \mathrm{eV}$ in work function at monolayer coverage. Flipped adsorption geometry on average (ethyl facing the surface) is found in the second layer with a $0.3 \mathrm{eV}$ increase in the work function. On ruthenium substrates the orientation of EC molecules could be controlled chlorine down or up by varying the oxygen coverage, as indicated by work function change measurements. $\triangle \mathrm{P}-\mathrm{TPD}$ from clean $\mathrm{Ru}(001)$ surface reveals a complete dissociation of the EC molecules on the clean $\mathrm{Ru}(001)$ surface at coverages up to $0.1 \pm 0.05 \mathrm{ML}$. At higher coverage it leads to two distinct TPD peaks: at $T=175$ and $120-130 \mathrm{~K}$, for the monolayer and multilayer coverage, respectively. Compression of preadsorbed 0.3 ML EC molecules into small islands on the surface occurs when ASW layers at coverage in the range $0.5-6$ bilayers (BL) are adsorbed on top of the EC covered surface, associated with a shift in peak desorption from $190 \mathrm{~K}$ down to $125 \mathrm{~K}$. A Caging process of EC molecules within the layers of ASW takes place by adsorbing more than $6 \mathrm{BL}$ until a fully caged system has developed above 20 ASW BL. A reversed TPD peak shift of the trapped EC molecules occurs from its compressed phase at $125 \mathrm{~K}$ up to $165 \mathrm{~K}$, the onset for ASW transition to crystalline ice and desorption. The detachment process leads to partial flipping over the geometry of the EC at the second layer, as determined by $\triangle \Phi$ measurement. The distance of the lifted and caged EC molecules from the Ru surface has been determined to reside $1.0 \pm 0.2 \mathrm{~nm}$ above the solid surface, based on the thermal dissociation and hydrogen uptake measurements of sandwiched EC molecules between a variable thickness ASW film and a fixed 10 BL layer, on top of clean $\mathrm{Ru}(001)$. We conclude that the caged EC molecules are trapped in a micelle-like geometry with the ethyl groups pointing inward. This conclusion is based on photodecomposition studies of the caged EC molecules at $193 \mathrm{~nm}$, as a function of EC initial coverage.
\end{abstract}

\section{Introduction}

Dipole-dipole interactions among neighbor adsorbates such as alkyl halides on surfaces have been the subject of intensive research in past years. ${ }^{1-4}$ This is partially due to their importance in the pesticide industry as well as their unique photochemistry and its damaging impact on the ozone layer. ${ }^{5}$

The first experimental demonstration of a molecular cage in amorphous solid water (ASW) was that of $\mathrm{N}_{2}{ }^{6}$ more than a decade ago. One of the main objectives of this study is the attempt to investigate how general the cage phenomenon is after being demonstrated for several molecules beside $\mathrm{N}_{2}$ such as $\mathrm{CCl}_{4},{ }^{7} \mathrm{CD}_{3} \mathrm{Cl}^{8},{ }^{8}$ and $\mathrm{CO}_{2} .{ }^{9}$

EC was chosen for this study not only as an extension of the family of cageable molecules to include longer chain alkyl halide, but also to investigate the photochemistry of this molecule while caged in ASW, and to characterize the photofragments. ${ }^{10}$

On the basis of previous studies,${ }^{11}$ the cage system seems to have a great influence on the outcome of photochemistry of trapped adsorbates on metal surfaces., ${ }^{9,10,12}$

A unique property of the cage is associated with blocking any photodesorption pathway, ${ }^{13,14}$ raising the probability of

\footnotetext{
† Part of the "Robert Benny Gerber Festschrift".

* To whom correspondence should be addressed.
}

photoreaction followed by rearrangement and formation of new molecular species. ${ }^{10,12}$

Another property of irradiated molecular cages is that the caged parent molecules and all irradiation products desorb simultaneously at the ASW crystallization with desorption onset near $165 \mathrm{~K}$. This fact significantly facilitates the search for new photoproducts.

Here we have focused on the determination of the actual location or distance of the caged molecules from the surface and the way these molecules assemble as a cage. This information is very important for a detailed understanding of the photochemical activity of adsorbates in general near metallic surfaces. ${ }^{10,15}$

The chemistry of alkyl chlorides reported in the literature indicates that these compounds are quite stable on metal surfaces at temperatures below $100 \mathrm{~K}$. Dissociation takes place for alkyl chains with $n_{\mathrm{c}} \geq 2$, with $n_{\mathrm{c}}$ being the number of carbon atoms in the alkyl chain, only at higher temperature to give adsorbed $\mathrm{C}_{n} \mathrm{H}_{x}$ fragments and $\mathrm{Cl}^{16-21}$

\section{Experimental Section}

The experiments described in this report were performed in an ultrahigh vacuum (UHV) apparatus, with a typical base pressure of $2 \times 10^{-10}$ Torr; details are given elsewhere. ${ }^{4,22}$

Briefly, a UHV chamber is equipped with a quadrupole mass spectrometer (QMS, VG SX-200) that is glass shrouded with a 

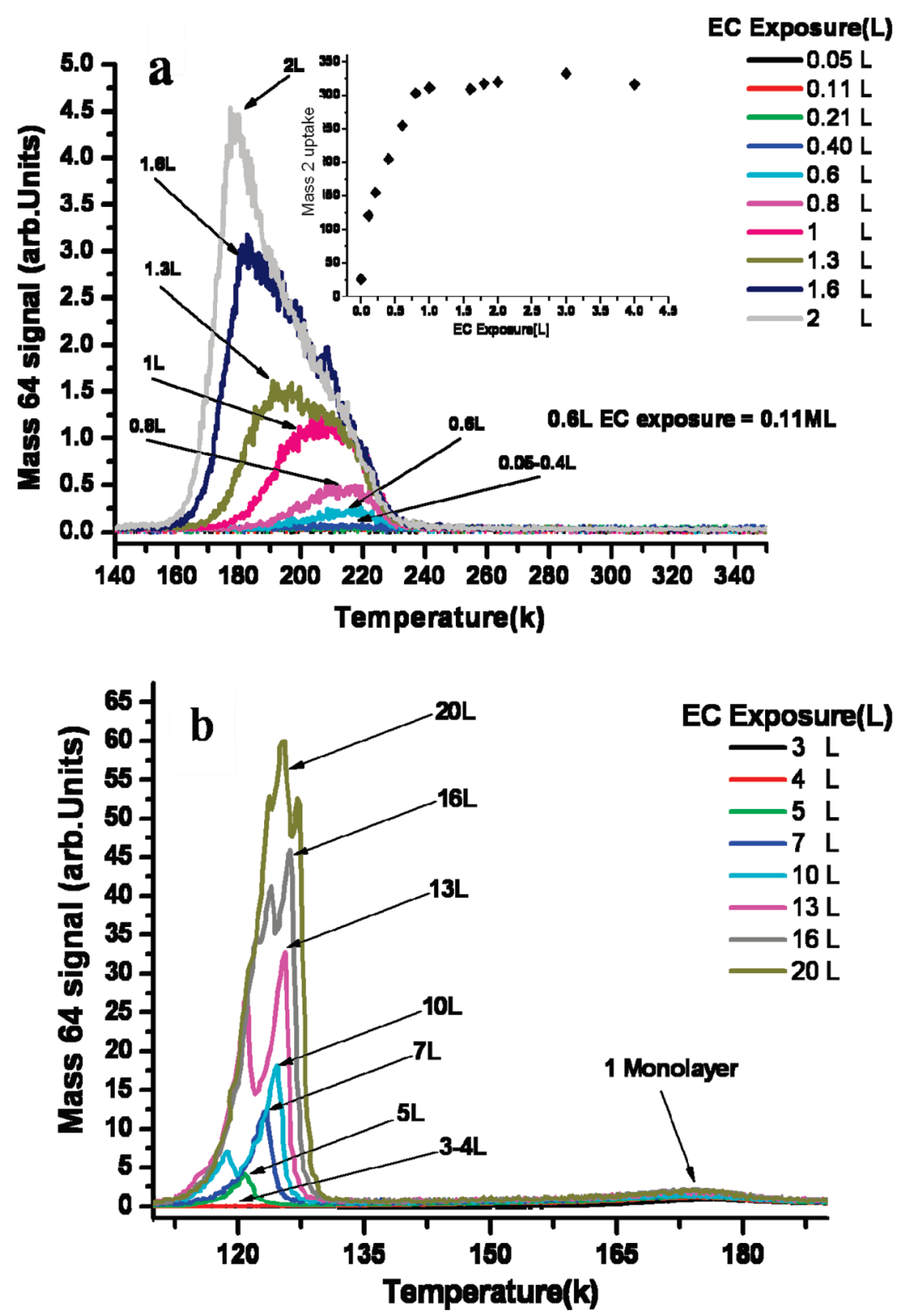

Figure 1. (a) $\triangle \mathrm{P}-\mathrm{TPD}$ spectra of $\mathrm{CH}_{3} \mathrm{CH}_{2} \mathrm{Cl}(\mathrm{EC})$ (m/e 64) at the indicated exposures $\left(1 \mathrm{~L}=10^{-6} \mathrm{Torr} \cdot \mathrm{s}\right)$ on clean $\mathrm{Ru}(001)$. The inset shows the resulting uptake of hydrogen as a function of EC exposure. (b) $\triangle \mathrm{P}-\mathrm{TPD}$ spectra of EC at the indicated higher exposures on clean Ru(001). The adsorption temperature was $90 \mathrm{~K}$ and the heating rate $2 \mathrm{deg} / \mathrm{s}$.

$3 \mathrm{~mm}$ aperture, avoiding recording desorption from surfaces other than the sample while improving sensitivity and selectivity. In addition to standard temperature programmed desorption $(\triangle \mathrm{P}-\mathrm{TPD})$, the average adsorbates dipole moment could be determined by means of a Kelvin probe (Besocke type $S$ ) that was employed to monitor the work function change $(\Delta \Phi)$, operated also in a $\Delta \Phi$-TPD mode as explained elsewhere. ${ }^{4}$

$\mathrm{The} \mathrm{Ru}(001)$ sample, oriented to within $0.5^{\circ}$ of the $(001)$ plane, could be cooled to $90 \mathrm{~K}$ by pumping over a liquid nitrogen reservoir. Temperature was determined by a C-type thermocouple (W5\%Re/W26\% Re) and controlled to within 0.5 deg by using an ac resistive heating LabView routine.

The crystal was cleaned by daily sputtering (Ar+ ions at 600 $\mathrm{V}$ and sample current of $\sim 6 \mu \mathrm{A}$ ) as well as oxidation at $850 \mathrm{~K}$ followed by annealing to $1620 \mathrm{~K}$ after each experiment. Daily calibration of the mass spectrometer sensitivity was performed by using fixed exposures equivalent to $1 \mathrm{ML}$ of the EC parent molecules (mass 64).
Exposure was preformed by backfilling the chamber through a leak valve to the desired pressure, measured in units of Langmuirs $\left(1 \mathrm{~L}=10^{-6}\right.$ Torr $\left.\bullet \mathrm{s}\right)$.

\section{Results and Discussion}

3.1. EC on Clean Ru(001). 3.1.1. Temperature Programmed Desorption ( $\triangle P$-TPD). Thermal desorption curves following EC adsorption on clean $\mathrm{Ru}(001)$ surface at $90 \mathrm{~K}$ are shown in Figure 1a,b.

No desorption of intact parent EC molecules could be detected at exposure below $0.5 \mathrm{~L}$ (equivalent to EC coverage of $0.1 \mathrm{ML}$ ) (Figure 1a) as a result of complete fragmentation. This is equivalent to a dissociation of $10( \pm 3) \%$ of the first EC monolayer on the Ru surface. Dissociating hydrocarbons are known to further decompose on clean ruthenium substrates, resulting in hydrogen evolution near $350 \mathrm{~K}$. The integrated hydrogen uptake obtained following exposure to the parent molecule is shown in the inset of Figure 1a. The inset reveals an increase of the hydrogen signal up to $0.6 \mathrm{~L}$. At this coverage 


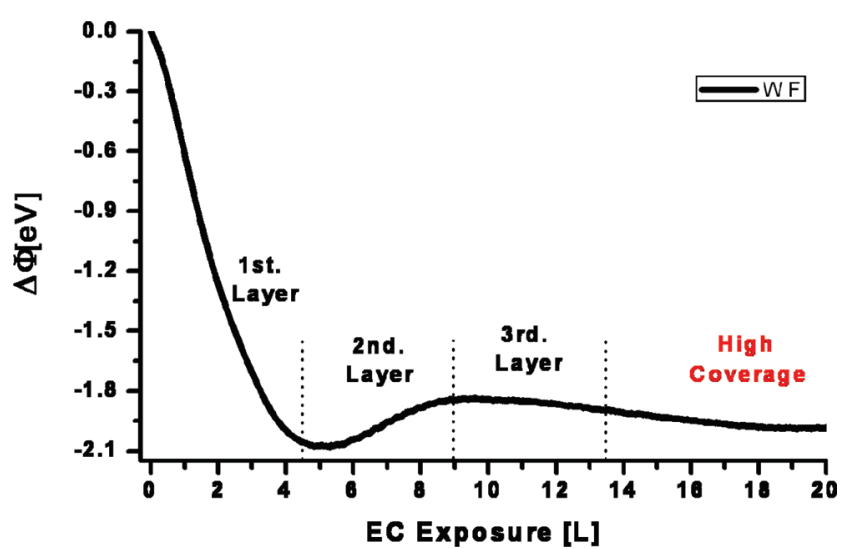

Figure 2. $\triangle \Phi$ measurement during EC adsorption at $82 \mathrm{~K}$ on $\mathrm{Ru}(001)$. The completion of each monolayer is marked by the vertical dashed lines.

onset of intact EC molecules TPD signal appears. At higher exposures (0.6 to $4.5 \mathrm{~L})$ a TPD peak appears at $170-180 \mathrm{~K}$ and saturates at $4.5 \mathrm{~L}$, indicating the completion of the first monolayer on $\mathrm{Ru}(001)$.

At a higher exposure a second peak emerges at $115-130 \mathrm{~K}$ that does not saturate, thus indicating it is part of the multilayer system. Within the multilayer peak one can distinguish the second, third, and fourth layer peaks every $\sim 4.5 \mathrm{~L}$ exposure of the EC molecules.

3.1.2. Work Function Change Measurements ( $\Delta \Phi)$. Initial adsorption at a low EC coverage leads to gradual decrease in the work function, continuously measured during the adsorption process with a Kelvin probe. The minimum is detected at $4.5-5$ $\mathrm{L}$, indicating the completion of the first monolayer, consistent with $\triangle \mathrm{P}-\mathrm{TPD}$ measurements discussed above, see Figure 1.

The $\triangle \Phi$ results shown in Figure 2 reveal a strong initial repulsive dipole-dipole interaction as all the EC molecules adsorb on average with chlorine toward the surface, resulting in a $\Delta \Phi$ decrease of $-2.1 \mathrm{eV}$. Repulsion among neighbor adsorbed EC molecules is confirmed by the broad $\triangle \mathrm{P}-\mathrm{TPD}$ peak (the monolayer peak) that stretches between 150 and $185 \mathrm{~K}$.

Further increase in coverage up to $2 \mathrm{ML}(9 \mathrm{~L})$ shows a turnover in the work function with an increase of $0.3 \mathrm{eV}$. This is based on the assumption that the sticking probability of EC on ruthenium is close to unity and is coverage independent. This suggests that the second layer has flipped adsorption geometry, on average, of most of the EC molecules, with the ethyl facing the $\mathrm{Ru}$ surface.

The flipping phenomenon goes on into the third layer where a smaller decrease in the work function $(\triangle \Phi=0.07 \mathrm{eV})$ is thought to be associated with a flipped geometry (chlorine down) once again.

Each turnover seen in the work function slope takes place near the completion of full layer, indicated also in the $\triangle \mathrm{P}-\mathrm{TPD}$ spectra in Figure 1b.

Similar adsorption behavior as seen in Figure 2 was previously discussed for $\mathrm{CD}_{3} \mathrm{Cl}^{8}$

On the basis of the Helmholtz equation $\left(\triangle \Phi=4 \Pi n_{i} u\right.$, where $n_{i}$ is the number of EC molecules in each of the first three layers $(i=1,2,3)$ that orient chlorine down $\left(n_{1}, n_{3}\right)$ or ethyl down $\left(n_{2}\right)$ and $\mu$ is the (fixed) dipole moment of an adsorbed EC molecule) one can extract the fraction of a monolayer of EC molecules that orient in either orientation. Assuming all EC molecules in the first layer orient (on average) the same way, namely chlorine down at the completion of the first monolayer, then $n_{2}=0.14 n_{1}$ and $n_{3}=0.034 n_{1}$.

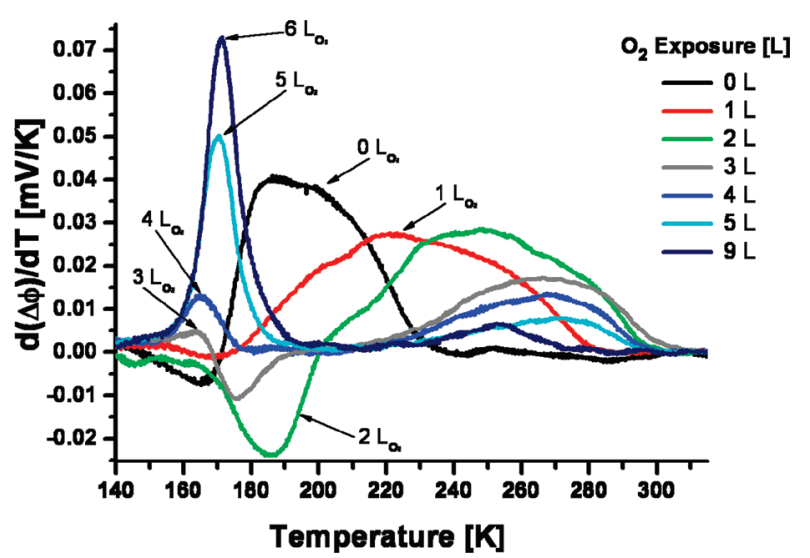

Figure 3. $\mathrm{d}(\triangle \Phi) / \mathrm{d} T$ of $\mathrm{EC}$ on a clean $\mathrm{Ru}(001)$ (thin black line) surface and oxygen-covered $\mathrm{Ru}$ at the indicated oxygen exposures $(4.5 \mathrm{~L}$ of oxygen $=0.25 \mathrm{ML}$ of $\mathrm{O} / \mathrm{Ru}$ ).

These results suggest that in the second layer there are $\sim 14 \%$ of a monolayer of EC molecules with Ethyl facing the surface, while in the third layer only $\sim 3 \%$ of the EC molecules reside with an opposite alignment of chlorine facing the surface before the molecules become randomly oriented in thicker layers.

3.2. Adsorbed Geometry of EC on $\mathrm{O} / \mathrm{Ru}$. The orientation of adsorbed EC molecules could be partially controlled chlorine down or up on $\mathrm{O} / \mathrm{Ru}(001)$ by tuning the oxygen coverage on the ruthenium surface.

At the second EC layer, reached by exposures of more than 4.5 L, EC molecules flip orientation as a result of local electrostatic effects caused by oxygen preadsorption.

Oxygen coverage of $\theta_{\text {oxy }}=0.13-0.25 \mathrm{ML}$ (exposures of 3-4.5 L) forces postadsorbed EC molecules to orient with chlorine up (ethyl toward the surface), while at oxygen coverage above $\theta_{\text {oxy }}=0.25 \mathrm{ML}$, the EC orientation flips over to the opposite adsorption geometry once again (chlorine down toward the surface).

This adsorbate geometry tuning is determined by monitoring $\triangle \Phi$ while ramping surface temperature in a $\triangle \Phi$-TPD mode. To observe the actual (average) direction of the molecular dipole a differential mode of detection, $\mathrm{d}(\triangle \Phi) / \mathrm{d} T$, is preformed as seen in Figure 3 A negative $\triangle \Phi$ change during TPD is the result of desorbing EC molecules that increase WF while residing in the second layer (total coverage $\theta_{\mathrm{EC}}=1.5 \mathrm{ML}$ ); this is observed at oxygen coverage of less than $0.25 \mathrm{ML}((2 \times 2) \mathrm{O} / \mathrm{Ru}(001)$ structure, reached at $4.5 \mathrm{~L}$ oxygen exposure).

At higher oxygen coverage, between $\theta_{\text {oxy }}=0.25$ and $0.5 \mathrm{ML}$, EC molecules lead to a decrease in the WF, therefore upon desorption an increase is recorded in the differential WF spectra in Figure 3.

The flipping of adsorbed dipolar molecules is observed only in the second layer, where the inter-EC molecules interaction is weak. It is not observed for submonolayer coverage since the EC-metal attraction in the first adsorbed layer is too strong and keeps the molecular orientation fixed. Similar behavior was previously reported in the case of $\mathrm{CD}_{3} \mathrm{Br}$ on $\mathrm{Ru}(001) .{ }^{23}$ It has been discussed in terms of a gradual increase of local dipole-dipole repulsion among neighbor EC molecules that is affected by the under laying oxygen covered ruthenium surface. At low oxygen density, second layer molecules can orient antiparallel to the first layer molecules, thus increasing the WF. However, at higher oxygen coverage, the overall work function of the $\mathrm{O} / \mathrm{Ru}$ substrate increases and more negative charge characterizes the top oxygen layer. This induces repulsion that flips the EC molecules toward a chlorine down configuration, 
leading to WF decrease and thus to a positive change in the differential spectra shown in Figure 3 for oxygen coverage $\theta_{\text {oxy }}>0.25 \mathrm{ML}$.

3.3. Interaction of EC with Coadsorbed Water. 3.3.1. Compression and Caging. $\triangle \mathrm{P}-\mathrm{TPD}$ spectra in Figure $4 \mathrm{~A}-\mathrm{G}$ reveal the gradual ethyl chloride cage formation while increasing ASW coverage. A fixed coverage of $0.3 \mathrm{ML}$ of EC was used throughout on clean $\mathrm{Ru}(001)$ surface, followed by the indicated water exposures.

Panels B-E of Figure 4 demonstrate the repulsion between water and EC molecules residing on the clean ruthenium surface. If compared to Figure 4A, where only clean EC desorbed (no water), one can clearly observe the significant shift to lower desorption temperature, as the ASW coverage increases. This is a result of compression of the EC molecules into local high coverage condensed islands.

A fully developed cage is obtained with a ASW layer thicker than $20 \mathrm{BL}$ (Figure $4 \mathrm{G}$ ) indicated by a narrow peak ( $5 \mathrm{~K}$ wide) at the onset of the ice crystallization and desorption (165 K).

The desorption peak emerging at $125 \mathrm{~K}$ (Figure 4D-F) coincides with TPD of EC molecules directly adsorbed on top of a $6 \mathrm{BL}$ thick layer of ASW (dashed line in Figure 4F).

This peak is attributed, therefore, to EC molecules that were lifted up by the accumulating water molecules from their original sites on the ruthenium surface to reside on top of the ASW layer.

Figure 5 reveals the apparent flipping of EC molecules that occurs upon coadsorption of water on top of EC on $\mathrm{Ru}$. Adsorption of $0.3 \mathrm{ML}$ of EC (1.5 L) leads to WF decrease of about $1 \mathrm{eV}$ (dashed line in Figure 5). At this point water molecules are introduced. Initially there is a further decrease of $0.6 \mathrm{eV}$ in the WF. Subsequent increase of ASW coverage, however, leads to a small increase of WF (about $0.15 \mathrm{eV}$ ), attributed to partial flipping of EC molecules. This is a similar effect discussed in section 3.1.2 at the second layer of pure EC on ruthenium, shown again in Figure 5 for comparison (dotted line). A possible explanation is that EC molecules are compressed by the coadsorbed water molecules, a process that leads to removal of strongly repelling EC molecules in condensed islands to a second layer. In the second layer the energetically more favorable adsorption geometry that minimizes dipoledipole repulsion is flipped molecules with the ethyl group facing the ruthenium surface. It is important to note that only a fraction of the EC molecules undergo this flipping, therefore the WF increase at total exposure $(\mathrm{EC}+$ water) of $5 \mathrm{~L}$ is only $0.15 \mathrm{eV}$.

Figure 6 presents a differential work function vs. temperature $\mathrm{d}(\triangle \Phi) / \mathrm{d} T$ spectra of the same stages shown as $\triangle \mathrm{P}-\mathrm{TPD}$ in Figure 4.

Upon adsorption of $0.4 \mathrm{BL}$ of water on top of $0.3 \mathrm{ML}$ of $\mathrm{EC}$, a new peak emerges in both the $\triangle \Phi$-TPD and $\triangle \mathrm{P}-\mathrm{TPD}$ at $160 \mathrm{~K}$, see Figure 4B. This peak is attributed to desorption from compressed islands of the EC molecules on the surface.

In the next stage seen in Figure 4C,D, the adsorption of 1BL of water forces the EC molecules to rearrange and form highly compressed islands. As a result of the repulsion between the compressed EC molecules, the TPD peak shifts to lower temperature $(135 \mathrm{~K})$ and the flipped over population grows as seen in Figures $4 \mathrm{C}$ and 6.

At higher water coverage (3-4 BL) a new peak, centered at $125 \mathrm{~K}$, emerges in the $\triangle \mathrm{p}$-TPD, Figure 4D,E, with no indication in the $\triangle \Phi$-TPD measurement, Figure 6 .

This peak has been attribution to EC molecules that were (partially) lifted away from the surface by the hydrogen bonded water to reside on top of the ramified ASW layers (Figure 4F).
At this ASW coverage (4 BL) there is an opposing effect on the WF: A fraction of EC molecules are still with chlorine down (lowering the WF) while these EC molecules that were lifted contributed the opposite change. Overall there is cancellation of the effect of both populations; therefore no change in the $\mathrm{WF}$ at $125 \mathrm{~K}$ is recorded.

A small negative $\mathrm{d}(\triangle \Phi) / \mathrm{d} T$ peak is observed at $125 \mathrm{~K}$ with $\theta_{\mathrm{ASW}}=6 \mathrm{BL}$ ( $6 \mathrm{~L}$ exposure), centered at $125 \mathrm{~K}$. It is attributed to lifted EC molecules onto the ramified, 3D and porous ASW layer. It turns out that a small fraction of these EC molecules do affect $\triangle \Phi$ in such a way that their desorption at $125 \mathrm{~K}$ results in the negative change seen in Figure 6. A similar small effect was consistently observed while adsorbing EC molecules on top of the preadsorbed ASW layer of $\theta_{\mathrm{ASW}}=6 \mathrm{BL}$ (not shown).

At water converge of $6 \mathrm{BL}$ a new $\triangle \mathrm{P}-\mathrm{TPD}$ peak appears centered at $160 \mathrm{~K}$ with no effect on WF measurements. This peak is attributed to the first encapsulated EC molecules but not fully caged. This new population cannot be seen in Figure $4 \mathrm{~F}$ (dotted curve) where we adsorb EC molecules on top of the water layer.

3.3.2. Distance of Caged EC Molecules from the Ruthenium Substrate. The question regarding the average distance of lifted up, caged EC molecules from the ruthenium substrate remained unresolved so far.

A lower limit estimate for the distance of caged molecules from the surface was determined in the following procedure, summarized in Figure 7.

The calibration approach has been to mimic the cage by constructing a sandwich of EC molecules between two films of ASW. This sandwich was prepared in two stages: Initially a variable thickness water layer was deposited on a clean $\mathrm{Ru}(001)$ at $90 \mathrm{~K}$. On top of that a fixed $0.3 \mathrm{ML}$ of EC was added (a unity sticking probability of EC on ASW is assumed) and finally a fixed $10 \mathrm{BL}$ of water was deposited to form the "sandwich cage". The thicker the first ASW layer is the further are the EC molecules from the ruthenium surface.

Hydrogen desorption ( $350 \mathrm{~K}$ peak) uptake has been used as a marker of EC molecules that reached the ruthenium surface upon desorption of the sandwiched $\mathrm{EC}-\mathrm{H}_{2} \mathrm{O}$ system.

One may summarize these experiments such that at ayer thickness of $9 \pm 1 \mathrm{BL}$ and above, the EC molecules do not reach the bottom ruthenium substrate during desorption of this sandwich composition and the amount of hydrogen desorbed from the surface is equal to that found when desorbing $10 \mathrm{BL}$ of water with no EC.

We conclude that the caged EC molecules must reside within a distance of at least $1.0 \pm 0.2 \mathrm{~nm}$ from the $\mathrm{Ru}$ surface.

3.4. EC@ASW: Cage Structure Derived from Photochemical Response. To gain an approximate picture of the way EC molecules assemble within the ASW film as a cage, we have performed photodecomposition experiments of the caged molecules. One may expect strong hydrophobic (repulsive) interactions between alkyl halide molecules and their surrounding water with increasing repulsion as the alkyl chain becomes longer. The chemical reaction between caged molecules following their photochemical decomposition has been chosen as a probe of their assembly and local structure, as a function of their initial EC coverage $\left(\theta_{\mathrm{i}, \mathrm{EC}}\right)$ prior to water postadsorption. We assume that the cage size is proportional to $\theta_{\mathrm{i}, \mathrm{EC}}$.

Once the EC-ASW cage has been established, therefore, it was irradiated by UV light at $193 \mathrm{~nm}$, photon energy of 6.4 $\mathrm{eV}$, at a fixed pulse energy of $3 \pm 0.5 \mathrm{~mJ}$ per pulse. More details on photochemistry experimental setup of caged molecules is given in ref 10. At $3 \mathrm{~mJ}$ pulse energy, EC molecules caged in 

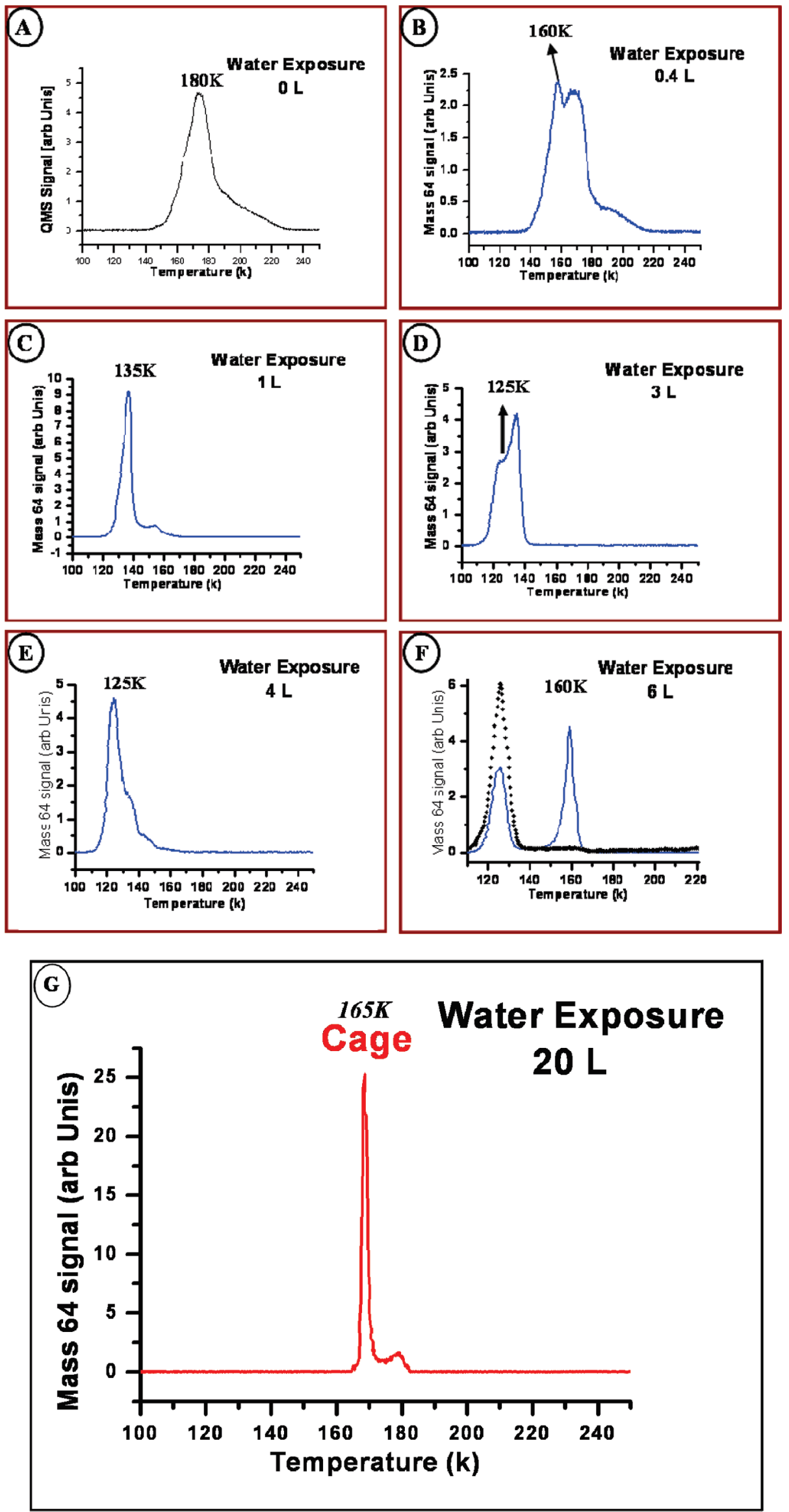

Figure 4. $\triangle \mathrm{P}$-TPD at mass 64 (parent EC molecules) following water postdeposition. Water coverage is indicated in Langmuirs $(1 \mathrm{~L}=1$ bilayer (BL)), on top of a fixed 0.3 ML of EC on clean Ru(001). The TPD spectra demonstrate the gradual (from A to E) compression and eventual full caging $(\mathrm{G})$ of EC under the indicated ASW layers. Note the substantially higher but narrower peak recorded following complete caging under 20 BL of ASW. The dotted line in panel F shows TPD spectra of postadsorption of $0.3 \mathrm{ML}$ of EC on top of $6 \mathrm{BL}$ of ASW. 


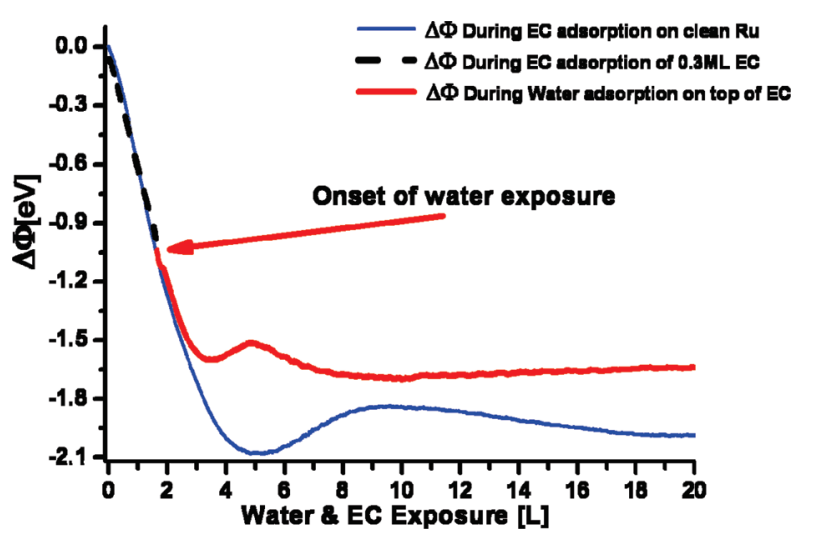

Figure 5. Work function change measurement during adsorption of EC on clean Ru (dotted line, as shown in Figure 2). Adsorption of EC molecules (dashed line up to $1.5 \mathrm{~L}$ ) followed by exposure to $\mathrm{H}_{2} \mathrm{O}$ (solid line from 2 to $20 \mathrm{~L}$ ).

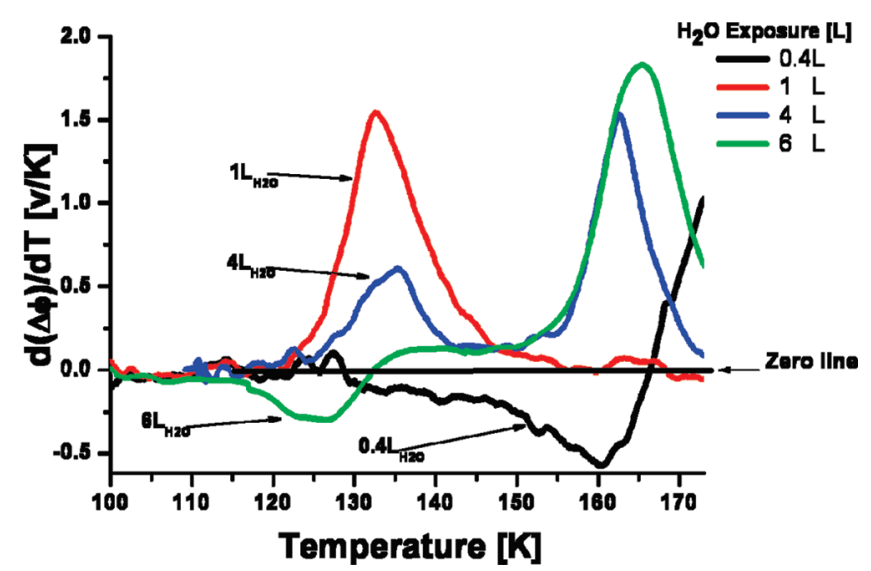

Figure 6. $\mathrm{d}(\triangle \Phi / \mathrm{d} T)$ of EC following water postdeposition on Ru. Obtained from the $\triangle \Phi$-TPD spectrum during desorption of EC. Two water exposures are emphasized $(0.4 \mathrm{~L}$ (black line) and $6 \mathrm{~L}$ (green line)) at which the EC molecules flip over (ethyl down).

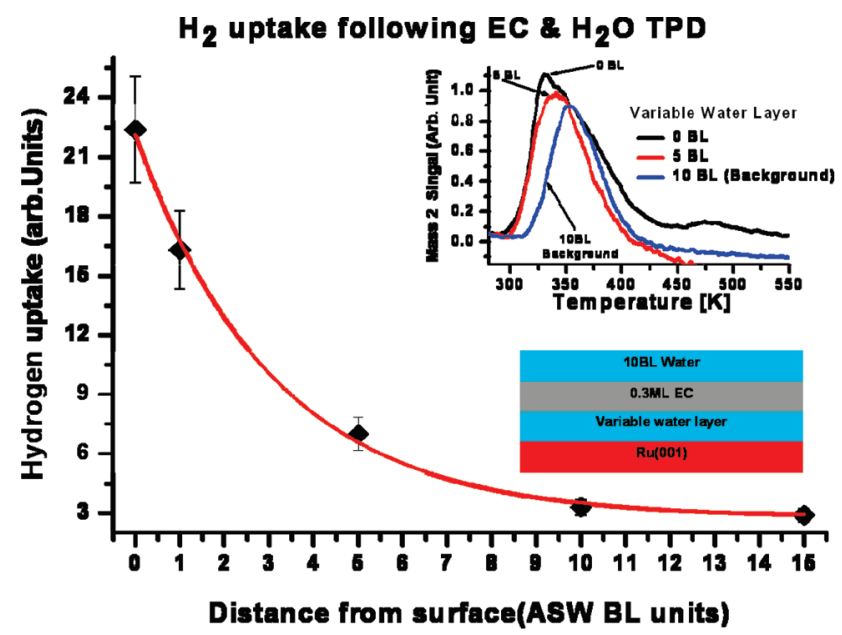

Figure 7. Uptake of desorbing hydrogen molecules following 0.3 ML of EC adsorption on the indicated water layer (variable) and covered by a fixed $10 \mathrm{BL}$ of water (fixed layer) on $\mathrm{Ru}(001)$. The line through the data points is a guide to the eye. The inset shows hydrogen TPD spectra obtained from EC molecules (no water), 5 BL of ASW away from the ruthenium surface and background $\mathrm{H}_{2}$-TPD from clean 10 $\mathrm{BL}$ of ASW. The heating rate was $2 \mathrm{deg} / \mathrm{s}$.

a matrix of ASW cannot undergo photodesorption. On the other hand, photofragments formed within the cage may react with each other but potentially also with the host water molecules surrounding the cage. An additional experimental benefit is

\section{EC@ASWIRu(001)}

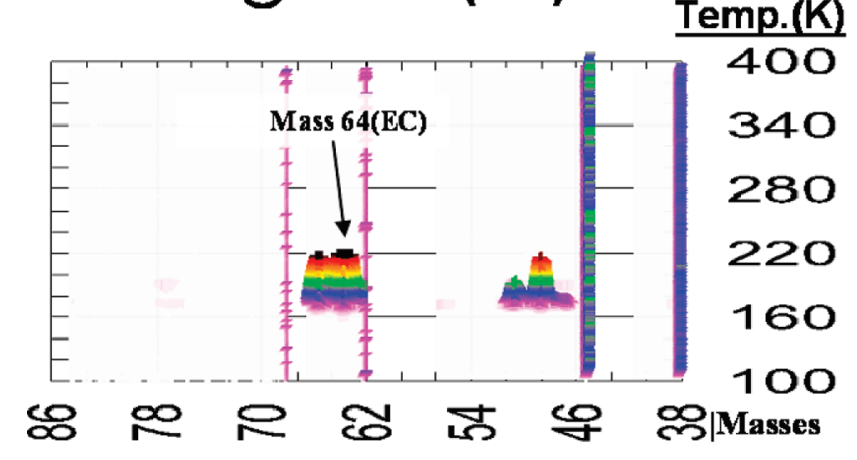

Irradiated EC@ASWIRu(001)

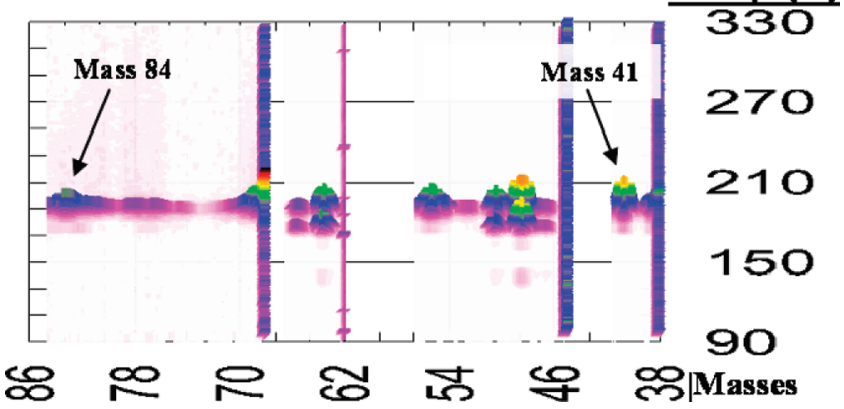

Figure 8. (A) A multimass-TPD simultaneously recorded at a range of masses between 38 and 85 au in a 3D representation without irradiation and (B) following $90 \mathrm{~s}$ of irradiation (equivalent to $5.2 \times$ $10^{18} 193 \mathrm{~nm}$ photons).

gained from the fact that the parent molecules as well as the various photoproducts are all explosively desorbing at the same temperature range near $165 \mathrm{~K}$, the onset of ASW desorption.

Postirradiation multimass $\triangle \mathrm{P}-\mathrm{TPD}$ spectra, shown in Figure 8 , simultaneously scan a complete mass range during a single TPD run for EC coverage.

Figure 8A shows a TPD scan of 2 ML of EC caged by 25 $\mathrm{BL}$ of $\mathrm{H}_{2} \mathrm{O}$, without irradiation. The signals seen in the plot other than masses 64 and 66 (parent molecule) arise from cracking of the parent molecule at the ionizer of the QMS.

Figure $8 \mathrm{~B}$ was obtained following the same EC cage system, after irradiation for $90 \mathrm{~s}$ (equivalent to a photon dose of $5.2 \times$ $10^{18}$ at $193 \mathrm{~nm}$ ). Postirradiation stable molecular products can be observed in the TPD scan at the mass range of 54-57, 39-43 and 75-84 amu.

EC molecules decomposing within the cage result in photoproducts that are exclusively short-chain hydrocarbons. The most abundant products $\left(\mathrm{C}_{4} \mathrm{H}_{8}, \mathrm{C}_{3} \mathrm{H}_{5}, \mathrm{C}_{3} \mathrm{H}_{3}, \mathrm{C}_{6} \mathrm{H}_{6}\right.$, and $\left.\mathrm{C}_{6} \mathrm{H}_{12}\right)$ do not include oxygen-containing molecules. Photoinduced decomposition of ethyl chloride molecules (EC) within a layer of amorphous solid water (ASW) on top of clean and oxygencovered $\mathrm{Ru}(001)$ has been described and fully analyzed elsewhere. ${ }^{4}$

Increasing the initial coverage of the parent EC molecules is expected to change the density and size of the cages formed within the ASW layer. The behavior following photodecomposition depends on the specific reaction mechanism for the formation of each of these photoproducts. For simplicity we assume that the cage system consists of spherical cages (bubbles), therefore higher EC coverage leads to larger spherical cages. The surface area of these cages (outermost layer or spheres) is proportional to $R^{2}$ ( $R$ is the average radius of the cages) and to the initial coverage of $\mathrm{EC}, \theta_{\mathrm{EC}}^{\mathrm{i}}$. The volume of 

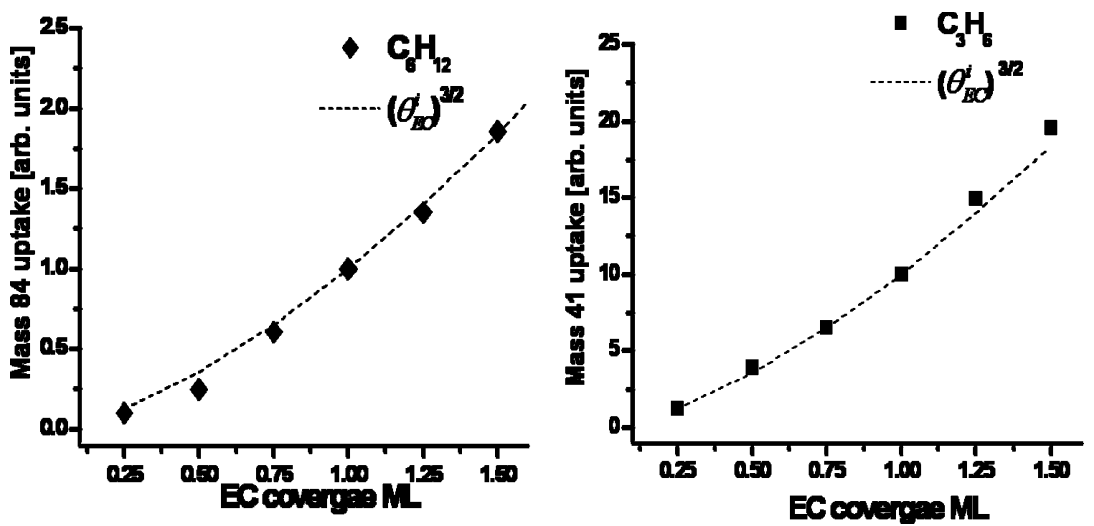

Figure 9. Dependence of the photoproducts' yield (filled symbols) on the initial EC molecules coverage $\left(\theta_{\mathrm{EC}}^{\mathrm{i}}\right)$. The dashed lines are calculated values based on the indicated dependence on the initial coverage of EC. Irradiation conditions were $90 \mathrm{~s}$ of irradiation (equivalent to $5.2 \times 10^{18}$ $193 \mathrm{~nm}$ photons).

cages would be proportional to $R^{3}$. Consequently, photoproducts that can only be formed at the core of the cage (photoproducts containing no oxygen) based on the reaction mechanism that requires direct contact with the surrounding $\mathrm{EC}$ caged molecules should follow a $\left(\theta_{\mathrm{EC}}^{\mathrm{i}}\right)^{3 / 2}$ dependence on initial coverage, as seen for two selected masses in Figure 9.

Unlike photochemistry of the $\mathrm{CD}_{3} \mathrm{Cl} @ \mathrm{ASW}$ system that produces different photoproducts including oxygen contacting ones, ${ }^{22}$ photochemistry of caged EC reveals various photoproducts none of which contains oxygen. This fact implies that all photoproducts in the EC system are core products, therefore follow the $\left(\theta_{\mathrm{EC}}^{\mathrm{i}}\right)^{3 / 2}$ dependence.

We may conclude that alkyl chains containing two carbons (e.g., $\mathrm{C}_{2} \mathrm{H}_{5}$ ) are long enough to form micelle-like cage structures (hydrocarbon chain inward, chlorine toward the surrounding water) to minimize hydrophobic interactions. This arrangement prevents reaction of ethyl radicals formed photochemically with the surrounding water molecules. In contrast, with shorter chains such as the case of $\mathrm{CD}_{3} \mathrm{Cl} @ \mathrm{ASW}$ where hydrophobic interactions are expected to be weaker, the reaction of photoproducts with the surrounding water has actually been reported. ${ }^{22}$

\section{Conclusions}

The interaction of ethyl chloride (EC) molecules on clean and oxygen-covered $\mathrm{Ru}(001)$ surfaces has been studied, leading to $12 \%$ dissociation at monolayer coverage on the clean ruthenium. Higher coverage is characterized by changing orientations, chlorine or ethyl facing the substrate. Orientation changes were observed vs. EC density at the second and third layers or by substrate oxygen coverage due to intermolecular electrostatic repulsion, as revealed by work function change measurements.

Adsorbing water on top of the EC covered surface leads to compression that results in significant decrease of desorption temperature from $250 \mathrm{~K}$ down to $135 \mathrm{~K}$. Further increase of the amorphous solid water (ASW) layer thickness leads to lifting the EC molecules away from the ruthenium surface with gradual formation of a cage within the ASW layer. The lifted and caged molecules do not affect work function measurements while explosively desorbing at the onset of ASW crystallization and desorption at $165 \mathrm{~K}$.

The position of the cage with respect to the ruthenium substrate has been estimated at $1.0 \pm 0.3 \mathrm{~nm}$. This estimate is based on the hydrogen uptake record of dissociating EC molecules residing within a gradually thickening sandwich of two ASW layers.

Finally, a micelle-like shape of the EC cage within ASW has been proposed, following its UV irradiation that has led to the hydrocarbon photofragments density increasing with initial EC coverage to the power of $3 / 2\left(\theta_{\mathrm{EC}}^{3 / 2}\right)$. This dependence suggests that the ethyl groups of caged EC molecules are all facing inward due to strong hydrophobic interactions with the surrounding water molecules.

Acknowledgment. We wish to thank Yigal Lilach for his valuable help in performiing this study. This research was partially supported by a grant from the German-Israel Foundation, US-Israel Binational Science Foundation, and the Israel Science Foundation. The Farkas center is supported by the Bundesministerium fur Forschung und Technologie and the Minerva Gesellschaft fur die Forschung mbh.

\section{References and Notes}

(1) Bent, B. E. Chem. Rev. 1996, 96, 1361.

(2) Berko, A.; Erley, W.; Sander, D. J. Chem. Phys. 1990, 93, 8300.

(3) Maschhoff, B. L.; Cowin, J. P. J. Chem. Phys. 1994, 101, 8138. 138.

(4) Livneh, T.; Lilach, Y.; Asscher, M. J. Chem. Phys. 1999, 111, 11-

(5) Rowland, F. S. Phil. Trans. R. Soc. B 2006, 361, 769-790.

(6) Livneh, T.; Romm, L.; Asscher, M. Surf. Sci. 1996, 315, 250.

(7) Smith, R. S.; Huang, C.; Wong, E. K. L.; Kay, B. D. Phys. Rev. Lett. 1997, 79, 909.

(8) Lilach, Y.; Asscher, M. J. Chem. Phys. 2002, 117, 6730-36.

(9) Berger, R.; Lilach, Y.; Ayoub, Y.; Asscher, M. Isr. J. Chem. 2005, $45,97$.

(10) Ayoub, Y.; Asscher, M. Phys. Chem. Chem. Phys. 2008, 10, 64866491.

(11) Dixon-Warren, St. J.; Heyd, D. V.; Jensen, E. T.; Polanyi, J. C. J. Chem. Phys. 1993, 98 (7), 5954.

(12) Lilach, Y.; Asscher, M. J. Chem. Phys. 2003, 119 (1), 407.

(13) Zhou, X.-L.; Zhu, X. Y.; White, J. M. Surf. Sci. Rep. 1991, 13, 73.

(14) Dai, H. L., Ho, W., Eds. Laser Spectroscopy and Photochemistry on Metal Surfaces, Parts I and II; World Scientific: New York, 1995.

(15) Zhu, X. Y. Annu. Rev. Phys. Chem. 1994, 45, 113.

(16) Zhou, X.-L.; White, J. M. Surf. Sci. 1988, 194, 438.

(17) Henderson, M. A.; Mitchell, G. E.; White, J. M. Surf. Sci. 1987, 184, L325.

(18) Chen, J. G.; Beebe, T. P., Jr.; Crowell, J. E.; Yates, J. T., Jr. J. Am. Chem. Soc. 1987, 109, 1726.

(19) Berkó, A. J. Phys. Chem. 1989, 93, 12.

(20) Zhou, X.-L.; Blass, P. M.; Cannon, K. C.; Solymosi, F.; White, J. M. Surf. Sci. 1989, 219, 294.

(21) Solymosi, F.; Kiss, J.; Révész, K. J. Phys. Chem. 1990, 94, 2224.

(22) Lilach, Y.; Asscher, M. J. Chem. Phys. 2003, 119, 407.

(23) Lilach, Y.; Asscher, M. J. Phys. Chem. B 2004, 108, 4358-4361.

JP900888J 